\title{
Molecular Complex of Lumiflavin and 2-Aminobenzoic Acid: Crystal Structure, Crystal Spectra, and Solution Properties ${ }^{\dagger}$
}

\author{
Huey-Sheng Shieh, ${ }^{\ddagger}$ Sandro Ghisla, ${ }^{\S}$ Louise Karle Hanson, ${ }^{\perp}$ Martha L. Ludwig, ${ }^{*}$ and Christer E. Nordman
}

ABSTRACT: The molecular complex lumiflavin-2-aminobenzoic acid monohydrate $\left(\mathrm{C}_{13} \mathrm{H}_{12} \mathrm{~N}_{4} \mathrm{O}_{2} \cdot \mathrm{C}_{7} \mathrm{H}_{7} \mathrm{NO}_{2} \cdot \mathrm{H}_{2} \mathrm{O}\right)$ crystallizes from aqueous solution as red triclinic prisms. The space group is $P \bar{I}$ with cell dimensions $a=9.660 \AA, b=14.866 \AA, c=$ $7.045 \AA, \alpha=95.44^{\circ}, \beta=95.86^{\circ}$, and $\gamma=105.66^{\circ}$. The crystal structure was solved by direct methods and refined by block-diagonal least-squares procedures to an $R$ value of 0.050 on the basis of 1338 observed reflections. The structure is composed of stacks of alternating lumiflavin and un-ionized (neutral) 2-aminobenzoic acid molecules. Two different modes of stacking interaction are observed. In one, 2-aminobenzoic acid overlaps all three of the isoalloxazine rings, at a mean distance of $3.36 \AA$; in the other, 2-aminobenzoic acid interacts with the pyrazine and dimethylbenzene moieties, at a distance of $3.42 \AA$. Perpendicular to the stacking direction, the mol-

I the presence of appropriate ligands, both model flavins and flavoproteins can form molecular complexes which absorb light in the wavelength region beyond $500 \mathrm{~nm}$. Flavoprotein complexes exhibiting distinctive long wavelength absorbance include complexes of NADH with NADH peroxidase (Dolin, 1957), of reduced lipoamide dehydrogenase with NAD ${ }^{+}$ (Massey \& Palmer, 1962), of reduced D-amino acid oxidase with imino acids (Massey \& Gibson, 1964; Yagi et al., 1967), and of D-amino acid oxidase with various substituted benzoates (Massey \& Ganther, 1965; Yagi et al., 1970). The properties of several such flavoprotein systems have been reviewed by Massey \& Ghisla (1974). The long wavelength bands in the absorption spectra of these systems are presumed to signal the formation of charge-transfer complexes. Charge transfer has been inferred from the frequency of the new band(s) and the simultaneous perturbation of the characteristic visible $\pi-\pi^{*}$ transitions of the isoalloxazine chromophore. However, this interpretation has been challenged in some cases, e.g., by Hemmerich \& Schuman-Jorns (1973), who attributed certain spectral modifications to formation of flavin-ligand $\sigma$ complexes. For the case of phenolate complexes of Old Yellow Enzyme, charge transfer has been documented by systematic correlation of the energy of the transition with the donor

\footnotetext{
${ }^{\dagger}$ From the Departments of Chemistry (C.E.N. and H.-S.S.) and Biological Chemistry (S.G. and M.L.L.), University of Michigan, Ann Arbor, Michigan 48109, and the Department of Energy and Environment (L.K.H.), Brookhaven National Laboratory, Upton, New York 11973. Received December 16, 1980. The work was supported by the National Institutes of Health (GM15259 and GM16429).

* Address correspondence to this author at the Biophysics Research Division, Institute of Science and Technology, The University of Michigan.

$\ddagger$ Present address: Physical Sciences Center, Monsanto Company, St. Louis, MO 63166.

Present address: Fachbereich Biologie, Universität Konstanz, Postfach 7733, D-775 Konstanz, West Germany.

$\perp$ Present address: Division of Chemical Sciences, Department of Energy and Environment, Brookhaven National Laboratory, Upton, NY 11973. Supported by the National Institute of Arthritis, Metabolism and Digestive Diseases of the National Institutes of Health and by the U.S. Department of Energy, Contract No. DE-AC-2-76CH00016.
}

ecules form a continuous sheet. Each flavin is hydrogen bonded via $\mathrm{O}(2)$ and $\mathrm{NH}(3)$ to two symmetrically related aminobenzoates; the water of crystallization forms three hydrogen bonds, bridging two flavins, via $O(4)$ and $N(5)$, and one aminobenzoic acid. The red color of the crystals results from a charge-transfer transition involving stacked flavin and 2-aminobenzoic acid molecules. Measurements of the polarized optical absorption spectra of crystals show that the transition moment direction for the long wavelength absorbance (beyond $530 \mathrm{~nm}$ ) contains an out-of-plane component which can only arise from a charge-transfer interaction. Since the amino $\mathbf{N}$ does not make exceptionally close interactions with isoalloxazine atoms in either stacking mode (minimum interatomic distance $3.52 \AA$ ), the charge transfer is presumed to involve $\pi$ orbitals of the 2 -aminobenzoic acid donor.

properties of various phenols and the reduction potential of several flavins (Abramowitz \& Massey, 1976). Demonstrations that certain long wavelength absorbing species can be intermediates in flavoprotein catalysis (Strickland \& Massey, 1973; Lockridge et al., 1972; Massey et al., 1970) have stimulated interest in the structures of these species.

Although no structures of flavoprotein charge-transfer complexes have been determined, crystallographic analysis of model compounds has established the geometries for hydroquinone-flavin (Bear et al., 1970; Kierkegaard et al., 1971), naphthalenediol-flavin (Langhoff \& Fritchie, 1970; Fritchie \& Johnston, 1975), tryamine-flavin (Inoue et al., 1980), and purine-flavin complexes (Scarbrough et al., 1976), all of which form highly colored crystals, indicating the presence of new transitions. These three-dimensional structures include face-to-face stacking interactions between isoalloxazine and ligand, suggesting that the characteristic absorbances arise from $\pi^{-} \pi^{*}$ charge transfer. However, transition moment directions for the long wavelength bands have not been measured in any of these crystalline model compounds.

When it binds to D-amino acid oxidase, 2-aminobenzoate gives rise to a new absorption band with a maximum near 550 $\mathrm{nm}\left(\epsilon=3130 \mathrm{M}^{-1} \mathrm{~cm}^{-1}\right)$. The resulting spectrum is typical of the "charge-transfer" complexes formed by flavoproteins (Massey \& Ganther, 1965; Massey \& Ghisla, 1974). For this reason we chose 2 -aminobenzoate as a ligand for model studies. The lumiflavin-2-aminobenzoic acid complex has been characterized by one of us (S.G.) and has been obtained in a crystalline form suitable for structure determination. Its spectrum in solution (Massey \& Ghisla, 1974) is similar to, though not identical with, the spectrum of the corresponding complex with D-amino acid oxidase (Massey \& Ganther, 1965)

This paper describes the crystal structure of the complex of lumiflavin (I), $\mathrm{R}=\mathrm{H}$, and 2-aminobenzoic acid and reports measurements of the polarized absorption spectra of single crystals in the 500-700-nm region. The dependence of the absorbance at wavelengths greater than $500 \mathrm{~nm}$ on crystal orientation provides direct evidence that the long wavelength 
<smiles>[R]n1c(=O)nc2n(C)c3cc(C)c(C)cc3nc-2c1=O</smiles>

absorption indeed corresponds to a charge-transfer transition involving stacked donor and acceptor molecules.

\section{Methods and Results}

Flavin-2-Aminobenzoic Acid Complexes in Solution. When high concentrations of 2-aminobenzoate are added to solutions of lumiflavin, a deep red color appears immediately, and under appropriate conditions (cf. below), red crystals precipitate. The solubility product, $K_{\mathrm{s}}=$ (lumiflavin)(2aminobenzoic acid), $\approx 3 \times 10^{-7}$ at $\mathrm{pH} 4$ and $24^{\circ} \mathrm{C}$. The difference spectra between complex and uncomplexed flavin at $\mathrm{pH} 7$ show positive maxima at 390 and $490 \mathrm{~nm}(+\Delta \epsilon=$ $100 \mathrm{M}^{-1} \mathrm{~cm}^{-1}$ ), a minimum at $\sim 435 \mathrm{~nm}$, and a long wavelength absorption extending well beyond $600 \mathrm{~nm}$ (Massey \& Ghisla, 1974). At pH 3.5, the difference spectra are qualitatively the same, but with the long wavelength tail extending only to $\sim 590 \mathrm{~nm}$ (Figure 1). Fluorescence emission is quenched in the complexes in the whole $\mathrm{pH}$ range investigated. $K_{\mathrm{d}}$ values of $0.1-0.2 \mathrm{M}$ were estimated from the difference spectra at $25^{\circ} \mathrm{C}$ and approximately constant ionic strength $(0.1 \mathrm{M} \mathrm{NaCl})$ by using $0.02 \mathrm{M}$ acetate, phosphate, and borate buffers at $\mathrm{pH} 3.5-9$ (cf. Figure 1). These $K_{\mathrm{d}}$ values are similar in magnitude to those found for a series of presumed flavin charge-transfer complexes (Tollin, 1968). We were prompted to study the $\mathrm{pH}$ dependence of $K_{\mathfrak{d}}$ because in the crystalline complex the 2-aminobenzoate is neutral, with the carboxyl group protonated and the amino group unprotonated. From the $\mathrm{pK}$ values of 2.03 and 4.98 (Cohn \& Edsall, 1943), one might expect the carboxyl group to ionize as the $\mathrm{pH}$ is increased. However, the observed $K_{\mathrm{d}}$ is essentially independent of $\mathrm{pH}$ in the range $\mathrm{pH} 3.5-9.0$, whether the flavin $\mathrm{N}(3)$ substituent $\mathrm{R}=\mathrm{H}, \mathrm{CH}_{2} \mathrm{COO}^{-}$, or $\left(\mathrm{CH}_{2}\right)_{3} \mathrm{SO}_{3}{ }^{-}$. (cf. structure I). Thus, if the anion does form, its charge does not significantly alter the stability of the complex in solution. On the other hand, $\mathrm{pH}$ affects the spectrum at long wavelengths (beyond $500 \mathrm{~nm}$ ). At high $\mathrm{pH}$, where 2-aminobenzoate should be ionized, the absorbance is increased almost 3-fold (Figure 1), suggesting that ionized 2-aminobenzoate is a better charge-transfer donor than the un-ionized species.

Preparation of Crystals of Lumiflavin-2-Aminobenzoic Acid. A solution $(10 \mathrm{~mL})$ of 2-aminobenzoic acid, slightly oversaturated at room temperature, was adjusted to $\mathrm{pH} 5.5$ and heated to $80-90^{\circ} \mathrm{C}$. A solution of lumiflavin (ca. 100 $\mathrm{mg} / \mathrm{mL}$ in $0.1 \mathrm{~N} \mathrm{NaOH}$ ) was added dropwise until crystallization began. The addition of the lumiflavin solution caused the $\mathrm{pH}$ to rise slightly. In the $\mathrm{pH}$ region $5.5-6.0$, precipitation of the red complex was fast, and no well-shaped crystals were obtained, so the first crystal crops were discarded by filtration. More lumiflavin was then added with an accompanying rise in $\mathrm{pH}$ of 0.2 to 0.3 unit, and crystals were again collected. The best crystals were obtained when the $\mathrm{pH}$ was between 6 and 7 and were washed with a little distilled water. An alternative method was to mix saturated solutions of lumiflavin and 2aminobenzoic acid, both at $\mathrm{pH} 4$, in a ratio of 3:1 to 5:1. Deep red crystals formed overnight at room temperature. When washed crystals were redissolved in water, the observed $\mathrm{pH}$ was about 4 , consistent with the presence in the crystal of the neutral species of aminobenzoic acid (see below).

Crystal and Powder Spectra. The polarized absorption spectra of single crystals of the lumiflavin-2-aminobenzoic acid

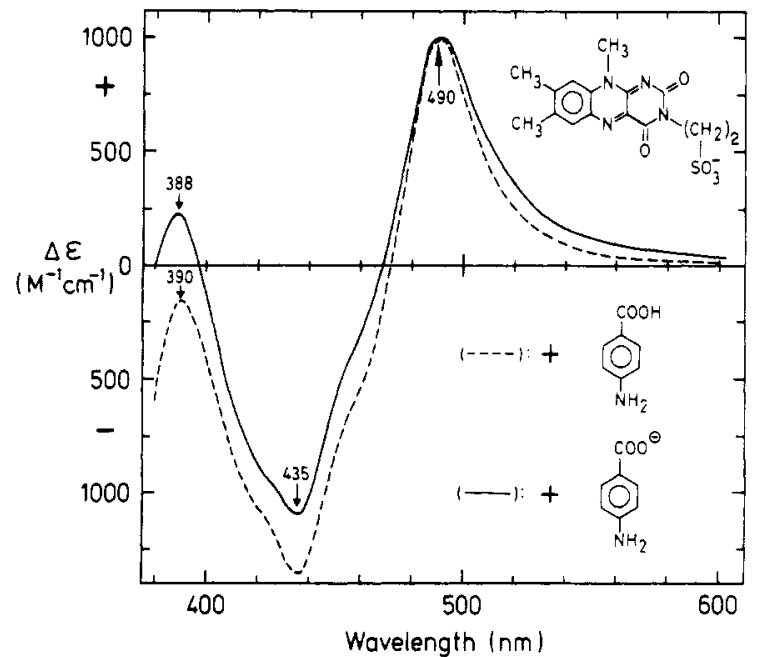

FIGURE 1: Perturbation of the flavin spectrum resulting from complexation with 2-aminobenzoic acid, as a function of $\mathrm{pH}$. Lumiflavin-3-propanesulfonate (insert), $2.0 \times 10^{-4} \mathrm{M}$ in $0.5 \mathrm{M} \mathrm{KCl}$, in either $0.02 \mathrm{M}$ citrate buffer (pH 3.5) (-.) or borate buffer ( $\mathrm{pH} 9.0$ ) $\left(-\right.$, was titrated with 2-aminobenzoate in the same buffer as $25^{\circ} \mathrm{C}$. Difference spectra were measured by using dual compartment cells $(0.45-\mathrm{cm}$ path length). The plotted values of $\Delta \epsilon$ were determined by extrapolation of double-reciprocal plots of the titration data.

complex were measured with a dual-beam recording microspectrophotometer (Eaton et al., 1975; Hofrichter, 1979). The crystals were placed on a glass cover slip on a rotating stage, and a given crystal was optically masked by imaging an adjustable field diaphragm onto the crystal plane. The spectra were collected with $32 \mathrm{X}$ Zeiss Ultrafluar Pol objectives. Due to the large extinction coefficients of the lumiflavin optical transitions, even the thinnest of the crystals grown in bulk solution was completely opaque to transmitted light below 500 $\mathrm{nm}$. Attempts to recrystallize the complex as thinner plates were unsuccessful. The crystals are thin laths, with the long edges always parallel to $c$. The well-developed faces are of two optically distinct types. The less dichroic of the two has been identified as the (110), and the best assignment for the more dichroic face is (110) (see below).

Powder absorption spectra of the crystalline complex and pure lumiflavin were obtained with a Cary $118 \mathrm{C}$ spectrophotometer interfaced with a Tektronix Model 4051 computer (Sutherland \& Boles, 1978; Sutherland et al., 1980). A thin film of powdered solid was sandwiched between a double layer of transparent gummed tape and placed immediately in front of the sample detector phototube to reduce scattering losses. The raw spectra were corrected for the tape base line and an analytical scattering function of the form $s=k \lambda^{-n}$.

Indexing of the Crystal Faces. To relate the projection of the transition moment to the crystal structure, it was essential to assign the Miller indices of the faces perpendicular to the incident beam of the microspectrophotometer. Crystals of a size adequate for indexing by $\mathrm{X}$-ray diffraction were too large for optical measurements, so separate crystals were used for each type of experiment. A single large lath with the same extinction directions and interedge angle $\left(73^{\circ}\right)$ as the crystal of Figure 5 was selected for indexing. When the major face was illuminated with polarized white light, this crystal varied in color from orange to deep red during rotation on the microscope stage, just as did the less dichroic face employed in the quantitative optical measurements. Preliminary precession photography suggested that this face was (110).

To confirm the indexing, we compared the crystal orientation required to reflect light from this face, using an optical goniostat, with a specific orientation satisfying the conditions 
Table 1: Crystal Data for Lumiflavin-2-Aminobenzoic Acid Monohydrate

$\begin{array}{ll}\text { formula unit } \mathrm{C}_{20} \mathrm{H}_{21} \mathrm{~N}_{5} \mathrm{O}_{5} & M_{\mathbf{r}} 411.22 \\ a=9.660(1) \AA & \text { space group } P \mathrm{I} \\ b=14.866(4) \AA & Z=2 \\ c=7.045(1) \AA & d_{\text {obsd }}=1.418 \mathrm{~g} \mathrm{~cm}^{-3} \\ \alpha=95.440(2)^{\circ} & d_{\text {calcd }}=1.421 \mathrm{~g} \mathrm{~cm}^{-3} \\ \beta=95.860(1)^{\circ} & \mu(\mathrm{Cu} \mathrm{K \alpha})=8.8 \mathrm{~cm}^{-1} \\ \gamma=105.660(2)^{\circ} & F(000)=432 \\ v=961.100(3) \AA^{3} & \end{array}$

for X-ray diffraction by the (110) plane on a four-circle diffractometer. On the optical goniostat, a face is brought into reflecting position by rotations $\omega_{\mathrm{g}}$ in the equatorial plane and $\phi_{g}$ about the spindle axis. The orientation matrix found on the diffractometer allows the calculation of many settings for diffraction from a given plane, but for comparison with the optical goniostat, $\chi$ must be fixed at $90^{\circ}$ and $2 \theta$ at $k$, where $k$ is a constant dependent on the zero point of the $\omega_{\mathrm{g}}$ scale. From the experimental orientation matrix, diffractometer settings $\omega_{d}$ and $\phi_{d}$ can then be calculated for diffraction from (1I0), which should be equivalent to the optical goniometer settings $\omega_{\mathrm{g}}$ and $\phi_{\mathrm{g}}$ for reflection from the less dichroic face. The calculated values were $\phi_{d}=252^{\circ}$ and $\omega_{d}=74.2^{\circ}$; the observed angles were $\phi_{\mathrm{g}}=249^{\circ}$ and $\omega_{\mathrm{g}}=75.3^{\circ}$. The results establish that the less dichroic face is (110).

The more dichroic face characteristically has an interedge angle of $69^{\circ}$ and changes in color from yellow to deep red as it is rotated on the stage of the microspectrophotometer while being illuminated with polarized white light. The extinction direction is $11^{\circ}$ from $c$ and $79^{\circ}$ from the short edge of this face. Crystals with well-developed "more dichroic" faces did not grow large enough for indexing by the above procedure. Precession photographs of crystals developed on (110) suggested that the highly dichroic face could be either (110) or (120). The (110) assignment is favored for several reasons. First, rotation of a large crystal, developed on (110), about $c$ shows that the major face is approximately $65^{\circ}$ from a view in which the crystal appears yellow; the calculated angle between perpendiculars to $(110)$ and $(110)$ is $65.10^{\circ}$, but the angle between ( 110$)$ and $(1 \overline{2} 0)$ is only $25.72^{\circ}$. Second, the measured interedge angle is within $1^{\circ}$ of the calculated value for the face (110) with edges [001] and [111]; comparable calculations for the $(1 \overline{2} 0)$ face disagree with the measured value of $69^{\circ}$ by more than $4^{\circ}$. Finally, the (110) assignment is consistent with the observed extinction direction, which should be approximately parallel to the isoalloxazine planes.

Structure Determination. Most crystals of a size appropriate for X-ray diffraction were found to be twinned (Darling, 1975). (Twins were readily recognized by examination with a polarizing microscope.) We were fortunate to obtain a single crystal of dimensions $0.15 \times 0.07 \times 0.04 \mathrm{~mm}$ by cutting a larger twin. Preliminary X-ray photographs revealed no symmetry other than Friedel symmetry, indicating that the crystal was triclinic. The density of the crystals, as determined by flotation in a $\mathrm{CCl}_{4} /$ benzene mixture, was $1.418 \mathrm{~g} / \mathrm{cm}^{3}$. This value agrees well with a calculated density of $1.421 \mathrm{~g} /$ $\mathrm{cm}^{3}$, based on two units of $\mathrm{C}_{13} \mathrm{H}_{12} \mathrm{~N}_{4} \mathrm{O}_{2} \cdot \mathrm{C}_{7} \mathrm{H}_{7} \mathrm{NO}_{2} \cdot \mathrm{H}_{2} \mathrm{O}$ per unit cell (Table I). The space group of the crystal was assumed to be $P \overline{1}$; this was confirmed by the successful refinement of the structure. Cell dimensions were calculated from a least-squares fit of 15 reflections with $2 \theta>50^{\circ}$.

Intensity measurements were made by using a Syntex $P \overline{1}$ diffractometer equipped with a graphite monochromator and employing $\mathrm{Cu} \mathrm{K} \alpha$ radiation. Reflection data were measured in the $\theta-2 \theta$ scan mode with a scan range of $2^{\circ}$, and with variable scan rate depending on the intensity of the reflection. A total of 2793 unique reflections within $2 \theta=120^{\circ}$ was collected. Of these, 1338 had intensities greater than 2.33 times their corresponding standard deviation $\sigma_{I}$ and were treated as observed data. The large number of "unobserved" reflections resulted from the small size of the crystal. Raw intensity data were corrected for Lorentz and polarization factors but not for absorption.

The intensity and $E$ distributions clearly revealed the hypercentric symmetry of the crystal which could be expected from the hexagonal arrangement of the atoms in both flavin and aminobenzoic acid. Despite the occurrence of several reflections with abnormally large $E$ values, e.g., $11 \overline{2}, 21 \overline{4}, 7 \overline{2} 2$, and $3 \overline{2} 6$ with $E$ values of $7.02,5.64,5.34$, and 5.04, respectively, the structure was readily solved with the MULTAN program (Main et al., 1974). The reason for the successful phasing was obvious from the convergence map; all reflections were linked so strongly through triplet phase relationships that a small group of strong reflections could not dominate. The phasing process used 499 high- $E$ reflections, 8 of which were assigned starting phases. Three of these eight were assigned a phase angle of $0^{\circ}$ to define the origin, one was given a known phase angle of $0^{\circ}$ from the $\sum$ relationship, and the remaining four were each given values of 0 or $180^{\circ}$. The resulting 16 phase sets were judged by ABSFOM (a measure of internal phase consistency), RESID (the discrepancy between observed data and calculated data), and PSIZERO (a test of phase correctness based on $E$-value agreement for weak relections not in the set). The phase set with the best PSIZERO, poorest ABSFOM, and second worst RESID turned out to be the correct one. The $E$ map based on this phase set revealed all 30 nonhydrogen atoms.

The structure was refined by block diagonal least-squares procedures. The quantity minimized was $\sum w\left\|F_{\mathrm{o}}|-k| F_{\mathrm{c}}\right\|^{2}$ where $F_{\mathrm{o}}$ and $F_{\mathrm{c}}$ are observed and calculated structure factors, respectively, $k$ is the overall scale factor, and $w$ is the weight of reflection $F_{0}$. The weighting scheme used in the refinement was $w=1 /\left(\sigma^{2}+\alpha F_{0}{ }^{2}\right)$, where $\sigma$ was the standard deviation associated with the structure factor $F_{0}$ and $\alpha$ was chosen so as to make the weighted discrepancy, $w\left\|F_{\mathrm{o}}|-k| F_{\mathrm{c}}\right\|^{2}$, as constant as possible in the entire range of $\left|F_{0}\right|$. Refinement of overall scale factor, all atomic coordinates, anisotropic thermal parameters of the 30 nonhydrogen atoms, and isotropic thermal parameters of the 21 hydrogen atoms reduced $R$ and $R_{w}\left[=\left(\sum w\left(\left|F_{\mathrm{o}}\right|-k\left|F_{\mathrm{c}}\right|\right)^{2} / \sum w F_{0}{ }^{2}\right)^{1 / 2}\right]$ to their final values of 0.050 and 0.048 , respectively, on the basis of 1338 observed reflections. In the last cycle of refinement, all parameter shifts were less than their corresponding estimated standard errors. A difference Fourier map using the parameters from the final cycle of refinement gave a maximum electron density of 0.19 $\mathrm{e} / \AA^{3}$ and revealed no significant features. Tables II and III contain the atomic positional and thermal parameters. The complete numbering system used in this study is shown in Figure 3. A table of observed and calculated structure amplitudes is available (see paragraph at end of paper regarding supplementary material).

Description of the Structure. The crystal structure consists of extended sheets of hydrogen-bonded planar molecules. These sheets pass through the center of inversion and coincide aproximately with the crystallographic $(11 \overline{2})$ planes. Figure 2 shows a portion of this molecular plane and the hydrogenbonding pattern in the structure. Figure 3 gives the atom labeling nomenclature. There are no direct flavin-flavin or benzoate-benzoate interactions in the structure. Each flavin is hydrogen bonded to two centrosymmetrically related ami- 
Table II: Positional and Thermal Parameters of Nonhydrogen Atoms

\begin{tabular}{|c|c|c|c|c|c|c|c|c|c|}
\hline name & $x$ & $y$ & $z$ & $\beta_{11}($ or $\beta)$ & $\beta_{22}$ & $\beta_{33}$ & $\beta_{12}$ & $\beta_{13}$ & $\beta_{23}$ \\
\hline$F N(1)$ & $0.4693(4)$ & $0.1136(2)$ & $-0.2041(5)$ & $76(6)$ & $86(2)$ & $237(12)$ & $10(3)$ & $-4(7)$ & $9(4)$ \\
\hline $\mathrm{FC}(2)$ & $0.3555(5)$ & $0.1464(3)$ & $-0.2575(7)$ & $90(7)$ & $89(3)$ & $231(15)$ & $7(4)$ & $-6(9)$ & $-1(5)$ \\
\hline FN(3) & $0.3686(4)$ & $0.2434(2)$ & $-0.2351(5)$ & $71(5)$ & $85(2)$ & $226(11)$ & $6(3)$ & $-16(7)$ & $4(4)$ \\
\hline $\mathrm{FC}(4)$ & $0.4915(5)$ & $0.3106(3)$ & $-0.1581(6)$ & $82(7)$ & $45(3)$ & $185(14)$ & $10(4)$ & $-1(8)$ & $0(5)$ \\
\hline$F C(4 a)$ & $0.6171(5)$ & $0.2765(3)$ & $-0.1025(6)$ & $74(7)$ & $88(3)$ & $160(13)$ & $11(3)$ & $-6(8)$ & $5(5)$ \\
\hline FN(5) & 0.7393 (4) & $0.3369(2)$ & $-0.0253(5)$ & $74(6)$ & $86(2)$ & $160(10)$ & $4(3)$ & $-13(6)$ & $-1(4)$ \\
\hline $\mathrm{FC}(5 \mathrm{a})$ & $0.8535(5)$ & $0.3038(3)$ & $0.0254(6)$ & $66(7)$ & $87(3)$ & 164 (13) & $12(3)$ & $20(8)$ & $18(5)$ \\
\hline $\mathrm{FC}(6)$ & $0.9857(5)$ & $0.3689(3)$ & $0.1077(6)$ & $91(7)$ & $49(3)$ & $163(13)$ & $18(4)$ & $15(8)$ & $4(5)$ \\
\hline $\mathrm{FC}(7)$ & $1.1048(5)$ & $0.3391(3)$ & $0.1625(6)$ & $80(7)$ & $55(3)$ & $158(13)$ & $10(4)$ & $2(8)$ & $-2(5)$ \\
\hline $\mathrm{FC}(8)$ & $1.0919(5)$ & $0.2414(3)$ & $0.1378(7)$ & $90(8)$ & $62(3)$ & $168(14)$ & $21(4)$ & $26(9)$ & $10(5)$ \\
\hline$F C(9)$ & $0.9645(5)$ & $0.1772(3)$ & $0.0559(7)$ & $109(8)$ & 49 (3) & $180(14)$ & $28(4)$ & $13(9)$ & $20(5)$ \\
\hline $\mathrm{FC}(9 \mathrm{a})$ & $0.8427(5)$ & $0.2077(3)$ & $-0.0005(6)$ & $75(7)$ & $41(3)$ & $140(12)$ & $11(3)$ & $-3(7)$ & $5(4)$ \\
\hline $\mathrm{FN}(10)$ & $0.7103(4)$ & $0.1446(2)$ & $-0.0800(5)$ & $85(6)$ & $38(2)$ & $165(11)$ & $16(3)$ & $4(6)$ & $14(4)$ \\
\hline$\Gamma C(10 a)$ & $0.5942(5)$ & $0.1761(3)$ & $-0.1330(6)$ & $94(7)$ & $40(3)$ & $145(12)$ & $14(4)$ & $5(8)$ & $15(5)$ \\
\hline $\mathrm{FO}(2)$ & $0.2355(4)$ & $0.0937(2)$ & $-0.3293(5)$ & $99(5)$ & $42(2)$ & $414(13)$ & $-1(3)$ & $-51(7)$ & $-12(4)$ \\
\hline FO(4) & $0.4980(4)$ & $0.3945(2)$ & $-0.1366(5)$ & $104(5)$ & $37(2)$ & $352(11)$ & $18(3)$ & $-42(6)$ & $-8(4)$ \\
\hline $\mathrm{FC}(7 \mathrm{a})$ & $1.2460(5)$ & $0.4101(4)$ & $0.2507(8)$ & $81(8)$ & $70(4)$ & $306(17)$ & $5(4)$ & $-32(10)$ & $-5(6)$ \\
\hline $\mathrm{FC}(8 \mathrm{a})$ & $1.2183(5)$ & $0.2053(4)$ & $0.2058(8)$ & $90(8)$ & $82(4)$ & $275(17)$ & $36(5)$ & $4(10)$ & $-10(6)$ \\
\hline $\mathrm{FC}\left(1^{\prime}\right)$ & $0.6938(6)$ & $0.0420(3)$ & $-0.1100(8)$ & $135(9)$ & $24(3)$ & $352(18)$ & $18(4)$ & $-8(10)$ & $17(5)$ \\
\hline $\mathrm{AC}(1)$ & $-0.1391(5)$ & $0.2669(3)$ & $-0.4752(6)$ & $75(7)$ & $43(3)$ & $124(12)$ & $14(4)$ & $8(7)$ & $-6(4)$ \\
\hline $\mathrm{AC}(2)$ & $-0.1857(5)$ & $0.1676(3)$ & $-0.5182(6)$ & $82(7)$ & $50(3)$ & $136(12)$ & $16(4)$ & $29(8)$ & $4(5)$ \\
\hline $\mathrm{AC}(3)$ & $-0.3322(5)$ & $0.1255(3)$ & $-0.5969(7)$ & $90(7)$ & $54(3)$ & $188(14)$ & $16(4)$ & $12(8)$ & $9(5)$ \\
\hline $\mathrm{AC}(4)$ & $-0.4241(5)$ & $0.1796(3)$ & $-0.6343(7)$ & $88(8)$ & $65(3)$ & $197(14)$ & $14(4)$ & $-20(9)$ & $-10(5)$ \\
\hline $\mathrm{AC}(5)$ & $-0.3784(5)$ & $0.2766(3)$ & $-0.5927(7)$ & $101(8)$ & $56(3)$ & $211(15)$ & $28(4)$ & $4(9)$ & $-2(5)$ \\
\hline$A C(6)$ & $-0.2358(5)$ & $0.3196(3)$ & $-0.5139(6)$ & $101(8)$ & $47(3)$ & $163(13)$ & $24(4)$ & $-15(8)$ & $11(5)$ \\
\hline $\mathrm{AC}(7)$ & $0.0106(5)$ & $0.3160(3)$ & $-0.3932(6)$ & $108(8)$ & $41(3)$ & $162(13)$ & $19(4)$ & $0(8)$ & $12(5)$ \\
\hline $\mathrm{AO}(1)$ & $0.0384(4)$ & $0.4088(2)$ & $-0.3560(5)$ & $100(5)$ & $37(2)$ & $311(11)$ & $6(3)$ & $-22(6)$ & $-3(3)$ \\
\hline $\mathrm{AO}(2)$ & $0.1068(3)$ & $0.2777(2)$ & $-0.3610(5)$ & $75(5)$ & $46(2)$ & $298(10)$ & $19(2)$ & $-27(6)$ & $3(4)$ \\
\hline AN & -0.0990 & $0.1108(2)$ & $-0.4811(6)$ & $100(6)$ & $39(2)$ & $296(13)$ & $25(3)$ & $-6(8)$ & $2(4)$ \\
\hline Wo & $0.3038(4)$ & $0.4981(2)$ & $-0.2185(5)$ & $124(6)$ & $45(2)$ & $327(11)$ & $16(3)$ & $-62(7)$ & $-22(4)$ \\
\hline
\end{tabular}

${ }^{a}$ The positional parameters are expressed as fractions of a unit cell edge. The anisotropic temperature factors have the functional form $T=\exp \left[-\left(h^{2} \beta_{11}+k^{2} \beta_{22}+l^{2} \beta_{33}+2 h k \beta_{12}+2 h l \beta_{13}+2 k l \beta_{23}\right) \times 10^{-4}\right]$. Standard deviations, as determined from the variance-covariance matrix of the final cycle of refinement, are given in parentheses and refer to the least significant digits of their corresponding parameters. The prefixes F. A, and $W$ refer to the lumiflavin, 2-aminobenzoic acid, and water molecules, respectively.

\begin{tabular}{|c|c|c|c|c|}
\hline name & $x$ & $y$ & $z$ & $B\left(\AA^{2}\right)$ \\
\hline FH(1) & $0.274(5)$ & $0.263(3)$ & $-0.285(6)$ & $2.7(1.1)$ \\
\hline $\mathrm{FH}(2)$ & $0.990(4)$ & $0.440(2)$ & $0.134(5)$ & $0.1(0.9)$ \\
\hline $\mathrm{FH}(3)$ & $0.952(4)$ & $0.107(3)$ & $0.049(5)$ & $1.7(1.0)$ \\
\hline $\mathrm{FH}(4)$ & $1.229(5)$ & $0.474(3)$ & $0.265(6)$ & $4.2(1.3)$ \\
\hline$\Gamma \dot{H}(5)$ & $1.326(6)$ & $0.400(3)$ & $0.181(7)$ & $6.3(1.6)$ \\
\hline FH(6) & $1.285(5)$ & $0.395(3)$ & $0.375(7)$ & $5.8(1.5)$ \\
\hline $\mathrm{FH}(7)$ & $1.200(5)$ & $0.144(3)$ & $0.146(6)$ & $3.8(1.2)$ \\
\hline $\mathrm{FH}(8)$ & $1,303(5)$ & $0.235(3)$ & $0.155(7)$ & $4.6(1.3)$ \\
\hline $\mathrm{FH}(9)$ & $1.257(7)$ & $0.221(4)$ & $0.361(9)$ & $11.7(2.3)$ \\
\hline $\mathrm{FH}(10)$ & $0.781(6)$ & $0.031(3)$ & $-0.166(7)$ & $6.2(1.6)$ \\
\hline FH(11) & $0.592(6)$ & $0.005(4)$ & $-0.200(8)$ & $7.9(1.8)$ \\
\hline $\mathrm{FH}(12)$ & $0.700(6)$ & $0.023(3)$ & $0.002(7)$ & $6.7(1.6)$ \\
\hline $\mathrm{AH}(1)$ & $-0.364(5)$ & $0.052(3)$ & -0. & 4.5 \\
\hline $\mathrm{AH}(2)$ & $-0.528(5)$ & $0.151(3)$ & $-0.684(5)$ & $1.9(1.1)$ \\
\hline $\mathrm{AH}(3)$ & $-0.442(5)$ & $0.318(3)$ & $-0.611(6)$ & $2.5(1.1)$ \\
\hline $\mathrm{AH}(4)$ & $-0.200(5)$ & $0.393(3)$ & $-0.486(6)$ & $3.4(1.2)$ \\
\hline $\mathrm{AH}(5)$ & $0.145(6)$ & $0.440(4)$ & $-0.299(7)$ & $6.5(1.6)$ \\
\hline $\mathrm{AH}(6)$ & $0.011(5)$ & $0.142(3)$ & $-0.438(6)$ & $4.2(1.3)$ \\
\hline $\mathrm{AH}(7)$ & $-0.128(5)$ & $0.043(3)$ & $-0.532(7)$ & $5.3(1.4)$ \\
\hline WH(1) & 0.369 & 0.4 & -0.1 & 9. \\
\hline WH(2) & $0.298(8)$ & $0.544(5)$ & $-0.113(9)$ & $13.3(2.5)$ \\
\hline
\end{tabular}

nobenzoates. The $O(2)$ of the lumiflavin is an acceptor for two amino hydrogens, one from each aminobenzoic acid molecule, while the flavin $\mathrm{NH}(3)$ is a donor to the carboxyl oxygen of aminobenzoic acid. The water of crystallization lies in the sheet, acting as a bridge between two flavins and one aminobenzoic acid (Figure 2).

The bond distances and angles in the lumiflavin molecule agree well with those determined in other crystal structures (Kierkegaard et al., 1971; Wang \& Fritchie, 1973; Trus et

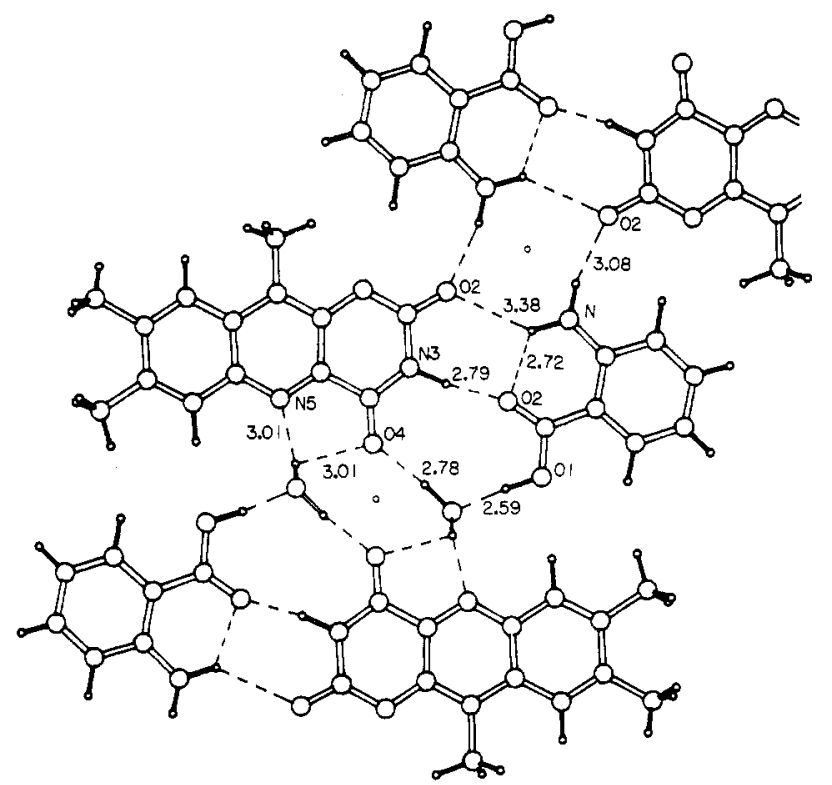

FIGURE 2: Drawing of the crystalline complex of lumiflavin-2. aminobenzoic acid viewed along an axis perpendicular to the planes of the isoalloxazine rings, illustrating the pattern of hydrogen bonding in the complex. Hydrogen bonds are indicated by dashed lines between the hydrogen and the acceptor atom. Hydrogen bond lengths are shown as donor-acceptor distances $(\AA)$. Centers of inversion are indicated as small circles.

al., 1971; Scarbrough et al., 1976). The structures of two crystal modifications of 2-aminobenzoic acid have been determined, a low-temperature modification (I) by Brown (1968) and a modification (II) stable above $81^{\circ} \mathrm{C}$ by Boone et al. 

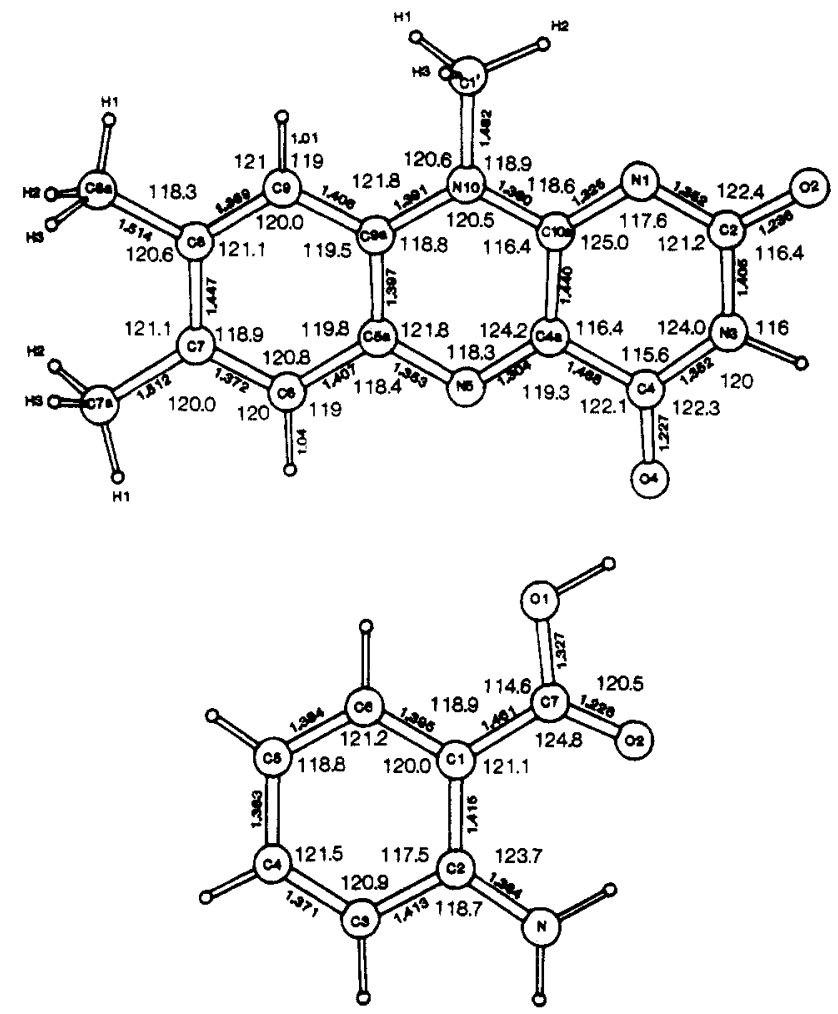

FIGURE 3: Covalent bond distances $(\AA)$ and angles (degrees) of the lumiflavin molecule (top) and the 2-aminobenzoic acid molecule (bottom). The estimated standard deviations of the nonhydrogen bond lengths and angles are $0.005 \AA$ and $0.4^{\circ}$, respectively.

(1977). In modification I, two independent molecules are present; one is in the neutral form and the other in the zwitterion form. Modification II contains neutral 2-aminobenzoic acid molecules, as does the complex reported here. The bond lengths and angles of the 2-aminobenzoic acid molecule in the complex agree well with those in modification II. The neutral molecule in modification I shows some apparently significant irregularity in the benzene ring geometry (Brown, 1968); this is not found in the present structure or in modification II.

In the present structure, both the lumiflavin and 2-aminobenzoic acid molecules are more closely planar, and the stacked donor and acceptor rings are more nearly parallel than in many comparable structures. Distortion of the isoalloxazine can be described as a bending about the line (N(10)-N(5). Typical values of this bending angle in oxidized uncomplexed flavins are $2.9^{\circ}$ for $N^{10}$-methylisoalloxazine (Wang \& Fritchie, 1973) and $1.8^{\circ}$ for $N^{3}$-methylisoalloxazine (Norrestam \& Stensland, 1972). In complexed flavins such as lumiflavin bromide-hydroquinone (Tillberg \& Norrestam, 1972), the value is $2.2^{\circ}$. The dihedral angle between the pyrimidinoid and benzenoid rings of the lumiflavin molecule in the present complex is only $0.7^{\circ}$. The mean planes of the stacked 2-aminobenzoic acid and lumiflavin rings in the complex structure are inclined at an angle of only $0.7^{\circ}$ whereas the angles between donor and acceptor rings in other flavin complexes are $2.3^{\circ}$ (Kuo et al., 1974), 3.6 (Tillberg \& Norrestam, 1972), and 4.1-7.7 (Scarbrough et al., 1976).

Interpretation of Polarized Absorption Spectra of Single Crystals. Polarized absorption spectra of oriented chromophores can reveal electronic transition moment directions. A $\pi \rightarrow \pi^{*}$ charge-transfer transition between stacked parallel donor and acceptor molecules will be directed along the intermolecular stacking axis, perpendicular to the molecular planes (Foster, 1969). Since parallel and antiparallel vectors

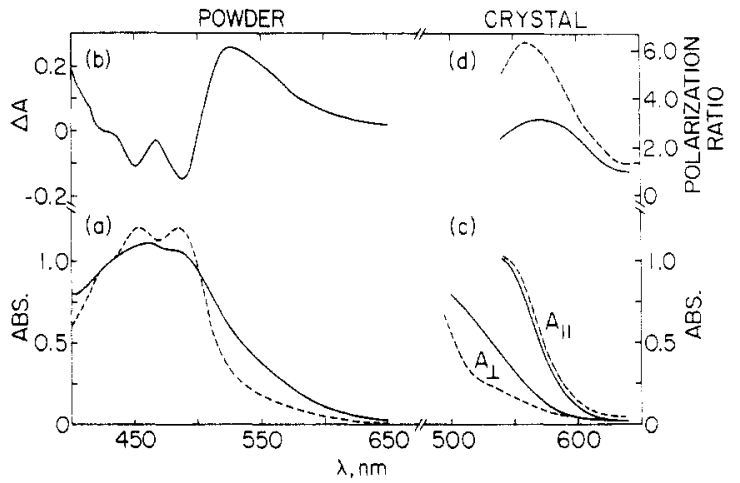

FIGURE 4: (a) Powder spectra of the lumiflavin-2-aminobenzoic acid complex (solid line) and pure lumiflavin (dashed line). (b) Complexed lumiflavin difference spectrum which emphasizes the increase in long wavelength absorption by the solid complex. (The parent spectra have been arbitrarily scaled.) (c) Polarized single crystal absorption spectra of the lumiflavin-o-aminobenzoic acid complex in the (110) (solid line) and (110) (dashed line) faces, designated $A_{\|}$and $A_{\perp} . A_{\|}$, the strongest absorbance, occurs for light polarized along the extinction axis which makes angles of $12.5^{\circ}[(110)]$ and $11^{\circ}[(110)]$ with the $c$ crystal axis. (d) Polarization ratio spectra, $\mathrm{PR}=A_{\|} / A_{\perp}$, for the (110) (solid line) and the (110) (dashed line).

are indistinguishable for electronic transitions, the $P \overline{1}$ lumiflavin-2-aminobenzoic acid crystals contain one unique stacking direction. The UV and visible electronic transitions of pure lumiflavin lie within the planes of the isoalloxazine rings, as shown by polarized absorption spectra of single crystals of flavodoxin (Eaton et al., 1975), polarized reflectance spectra of single crystals of a $\mathrm{Cu}$ (II) complex of 10-methylisoalloxazine (Yu et al., 1976), and electric linear dichroism and fluorescence polarization measurements on flavin mononucleotides in membranes (Johansson et al., 1979). An outof-plane component to in-plane transition dipoles cannot be induced by exciton coupling between parallel chromophores. Thus any out-of-plane polarization observed for crystals of the complex can be attributed to charge-transfer transition(s) between the 2-aminobenzoic acid and the lumiflavin.

Due to the thickness of the crystals, only the long wavelength region (above $500 \mathrm{~nm}$ ) was accessible to analysis. The powder spectra given in Figure 4 show that the lumiflavin-2-aminobenzoic acid solid has enhanced absorption beyond $500 \mathrm{~nm}$ relative to the pure lumiflavin solid, with the maximum difference occurring at about $530 \mathrm{~nm}$. These powder spectra are similar to solution spectra except for a red shifting of the visible wavelength transitions, probably because of exciton interactions between the closely packed chromophores of the solid.

The orientations of the optic axes of triclinic systems are free to vary with wavelength. Fortunately, the extinction axis dispersion of the lumiflavin-2-aminobenzoic acid crystals is less than $\pm 2^{\circ}$ from 530 to $700 \mathrm{~nm}$. Both of the two optically distinct crystal faces had extinction axes nearly parallel and perpendicular to the long edge of the parallelepiped, which corresponds to the $c$ axis of the crystal. The strongest absorption occurred along the extinction axis which was "parallel" to $c$. The polarized absorption spectra and polarization ratios for the less dichroic (110) and more dichroic (110) faces are shown in Figure 4. Maximum polarization ratios (PR), where $\mathrm{PR}=A_{\|} / A_{\perp}$, are 6.3 for the more dichroic face and 3.1 for the less dichroic face, at $560 \mathrm{~nm}$.

First, we show that the absorption beyond $530 \mathrm{~nm}$ must include a contribution from out-of-plane transition(s). In Figure $5, u$ is the more strongly absorbing extinction axis, $s$ is the stacking axis vector, and $c$ is the $c$ axis. The projections of these axes on the (110) face are shown in the figure and values for the angular relations between these directions, $\theta_{c w}$, 
Table IV: Angles Relating Optical and Crystallographic Directions

\begin{tabular}{|c|c|c|c|c|c|c|c|c|}
\hline \multirow[b]{2}{*}{ face } & \multirow[b]{2}{*}{$\theta_{c u}^{a}$} & \multirow[b]{2}{*}{$\theta_{c s}^{b}$} & \multirow[b]{2}{*}{$\theta_{s u}=\theta_{c u} \pm \theta_{c s}$} & \multirow[b]{2}{*}{$\theta_{t u}^{c}$} & \multicolumn{2}{|c|}{$\theta_{x c}{ }^{d}$} & \multicolumn{2}{|c|}{$\theta_{x u}=\theta_{x c} \pm \theta_{c u}$} \\
\hline & & & & & $\mathrm{E}^{e}$ & $\mathrm{~J}^{e}$ & $E^{e}$ & $\mathrm{j}^{e}$ \\
\hline $\begin{array}{l}1 \overline{10} \\
110\end{array}$ & $\begin{array}{l}12.5^{\circ} \\
11^{\circ}\end{array}$ & $\begin{array}{l}9.57^{\circ} \\
7.54^{\circ}\end{array}$ & $22.1^{\circ}, \frac{2.9^{\circ}}{3.5^{\circ}}$ & $\begin{array}{l} \pm 29.6^{\circ} \\
\pm 21.7^{\circ}\end{array}$ & $\begin{array}{l}52.1^{\circ} \\
68.5^{\circ}\end{array}$ & $\begin{array}{l}14.1^{\circ} \\
77.0^{\circ}\end{array}$ & $\begin{array}{l}\frac{64.6^{\circ}}{79.5^{\circ}}, 39.6^{\circ} \\
57.5^{\circ}\end{array}$ & $\begin{array}{l}26.6^{\circ}, \frac{1.6^{\circ}}{68.0^{\circ}, \frac{6.0^{\circ}}{}} \\
8\end{array}$ \\
\hline
\end{tabular}

${ }^{a} \theta_{c u}=$ angle between the $c$ axis and the $u$ extinction axis; measured optically. ${ }^{b} \theta_{c s}=$ angle between $s$, the projection of the stacking axis, and $c$; calculated from crystal coordinates. ${ }^{c} \theta_{t u}=$ angle between $t$, the projected transition moment direction of a "single" transition at $560 \mathrm{~nm}$, and $u .{ }^{d} \theta_{x c}=$ angle between $x$, the projection of the $445-\mathrm{nm}$ flavin transition moment direction, and $c$. $e E$ is the transition moment orientation of Eaton et al. (1975). Yu et al. (1976) agree to within $5^{\circ}$. $x$ was calculated from the $\mathrm{C}(8)-\mathrm{N}(3)$ vector, which makes an angle of $13.5^{\circ}$ with the long axis of the flavin ring. J is the orientation of Johansson et al. (1979) approximated as a vector from $O(4)$ to a point between $C(8)$ and $C(9)$, which makes an angle of $34.6^{\circ}$ with the long axis of the flavin.

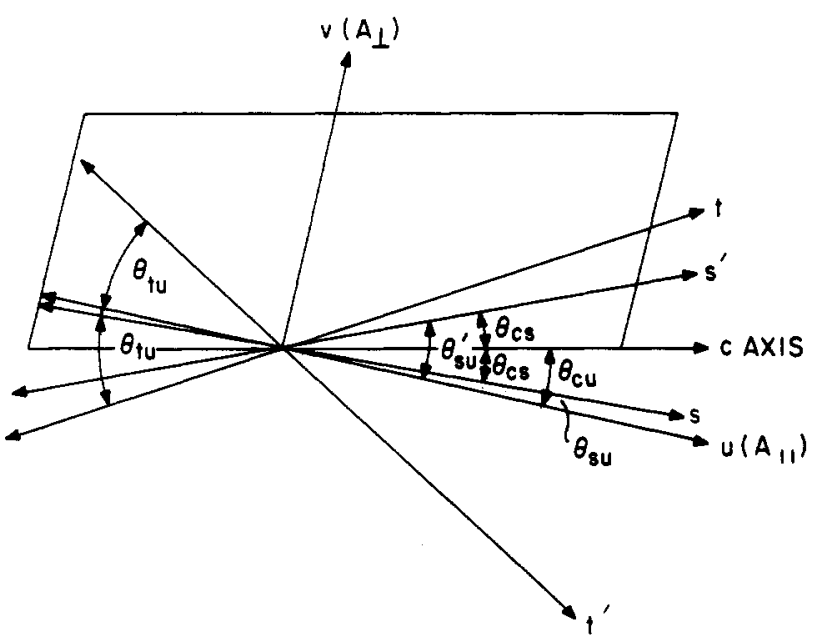

FIGURE 5: Lumiflavin-2-aminobenzoic acid (1 10 ) face whose long edge corresponds to the $c$ crystal axis. $u$ and $v$ are the extinction direction, $s$ and $s^{\prime}$ are the two possible orientations of the intermolecular stacking axis with respect to $c$, and $t$ and $t^{\prime}$ are the two possible orientations of the calculated transition dipole moment relative to the extinction axes, assuming a single transition at $560 \mathrm{~nm}$.

$\theta_{c s}$, and $\theta_{s u}$, are given in Table IV for both faces. Note that when the indices of the short edge of the crystal face are unknown, there will be an ambiguity in $s$ equivalent to a $180^{\circ}$ rotation of the crystal about $c$; the alternative vector is designated $s^{\prime}$.

If all the absorbance beyond $530 \mathrm{~nm}$ is due to a single transition, $\theta_{t u}$, the angle between the transition dipole moment direction $t$ and the extinction axis $u$ can be obtained from

$$
\mathrm{PR}=\frac{A_{\|}}{A_{\perp}}=\frac{\cos ^{2} \theta_{t u}}{\sin ^{2} \theta_{t u}}
$$

There is a 2-fold ambiguity of $t$ relative to $u$ (Figure 5). For the (110) face, $\theta_{t u}= \pm 29.6^{\circ}$, and for the (110) face, $\theta_{t u}=$ $\pm 21.7^{\circ}$.

Considering both $s$ and $s^{\prime}$, we have four choices for $t$ relative to $s$ in a given face. The closest approach of $t$ to $s$ is $7.5^{\circ}$ for the (110) face and $3.2^{\circ}$. for the (110) face. The largest possible deviations of $t$ (or $t^{\prime}$ ) from $s$ (or $s^{\prime}$ ) are $51.7^{\circ}$ and $40.2^{\circ}$, for the (110) and (110) faces, respectively. Does this "worst case" permit the $>530$-nm absorption to be explained entirely in terms of an in-plane transition, i.e., the long wavelength tail of the lowest energy $\pi \rightarrow \pi^{*}$ transition of the flavin? Two differing orientations for the dipole moment direction, $x$, of this transition have been proposed. The angles which both suggested orientations make with $c$ and $u, \theta_{x c}$ and $\theta_{x u}$, are given in Table IV (cf. Figure 6). The (110) data are clearly incompatible with either the Eaton et al. (1975) or the Johansson et al. (1979) choices for the in-plane transition. Hence the
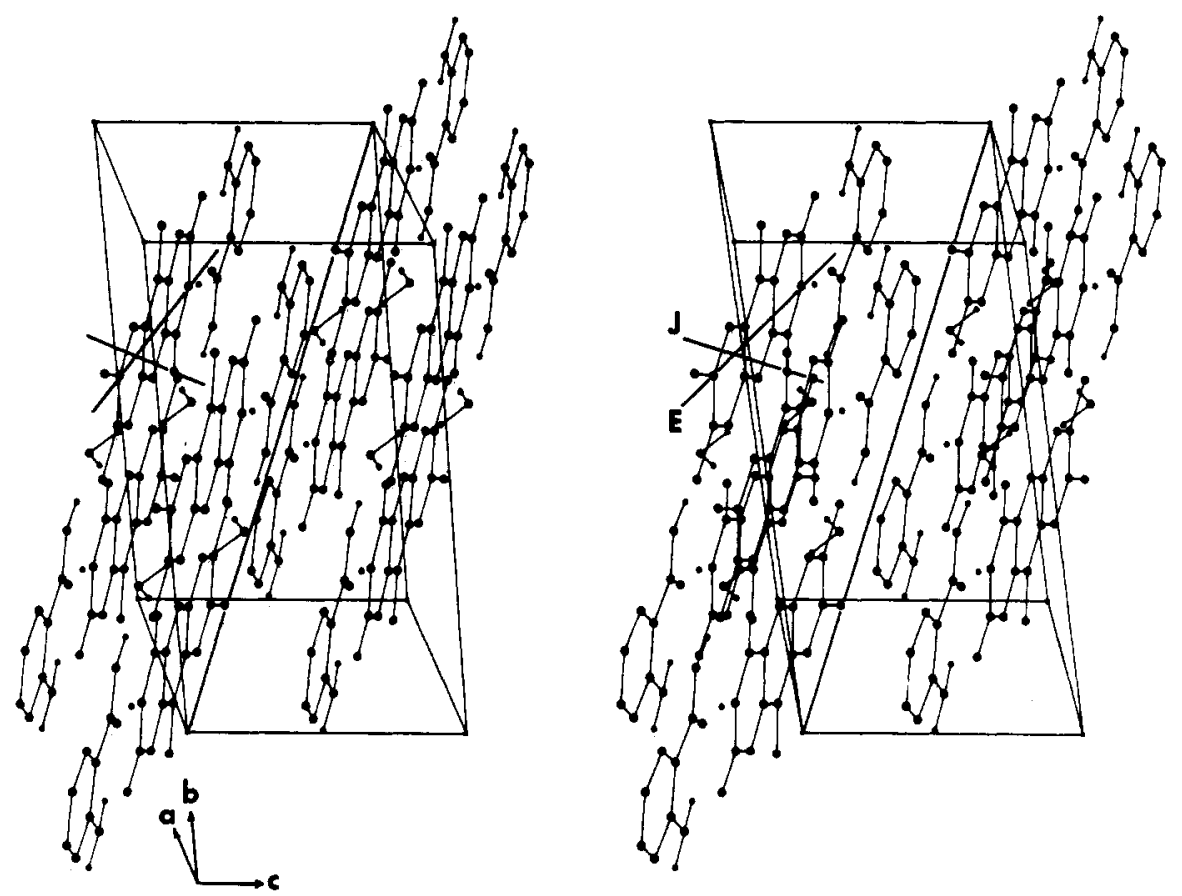

FIGURE 6: Stereo drawing showing the packing of lumiflavin-2-aminobenzoic acid in the unit cell. The view is perpendicular to the (110) face in an orientation corresponding to the diagram of Figure 5. The body diagonal [111] is the short edge of the crystal used in the spectroscopic experiments. Transition moment directions for the in-plane flavin transition near $450 \mathrm{~nm}$ are designated $E$ for the assignment of Eaton et al. (1975) and $J$ for the assignment of Johansson et al. (1979). 
$>530$-nm absorption contains an out-of-plane component, even with the least favorable interpretation of the polarization ratio data.

In fact, accessory data can be used to resolve the ambiguity in $s$. For each face, the choice of one alternative makes the stacking vector essentially coincident with the extinction axis $u$, as expected for a structure composed of planar networks. The angle $\theta_{s u}$ is then $2.9^{\circ}$ for the (110) face (Figure 6) and $3.5^{\circ}$ for the (110) face. Indexing of the short edge of each face confirms these choices. For ( 110$)$ the interedge angle establishes that the short edge corresponds to the body diagonal, [111]. This edge is nearly parallel to the flavin planes (Figure 6) and approximately perpendicular to the extinction direction, $\bar{u}$ (Figure 5). The short edge of the (110) face is similarly found to be [111]. The largest deviations of $t$ (or $t^{\prime}$ ) from $s$ are then $32.5^{\circ}$ for (110) and $25.2^{\circ}$ for (110).

Because the closest approach to $t$ to $s$ (as assigned above) is $26.7^{\circ}$ for $(110)$ and $18.2^{\circ}$ for $(110)$, we now consider a model in which the charge-transfer transition, expected to be parallel to $s$, contributes only part of the absorbance beyond $530 \mathrm{~nm}$. Since the power spectrum of the pure flavin reveals nonzero absorbance beyond $530 \mathrm{~nm}$, the observed polarization may arise from two perpendicular overlapping transitions, the in-plane flavin $\pi \rightarrow \pi^{*}, x$, and the out-of-plane charge transfer, $z$. The expression for the polarization ratio becomes

$$
\mathrm{PR}=\frac{A_{\|}}{A_{\perp}}=\frac{\epsilon_{x} \cos ^{2} \theta_{x u}+\epsilon_{z} \cos ^{2} \theta_{z u}}{\epsilon_{x} \sin ^{2} \theta_{x u}+\epsilon_{z} \sin ^{2} \theta_{z u}}
$$

where $\epsilon_{x}$ and $\epsilon_{z}$ are the molar extinction coefficients for the $x$ and $z$ transitions (Hofrichter \& Eaton, 1976). With data from two crystal faces, the internal consistency of various choices for $x$ and $z$ can be tested by using

$$
\frac{1-R \cos ^{2} \theta_{x u}}{1-R^{\prime} \cos ^{2} \theta_{x u}^{\prime}}=\frac{R \cos ^{2} \theta_{z u}-1}{R^{\prime} \cos ^{2} \theta_{z u}^{\prime}-1}
$$

where $R=(\mathrm{PR}+1) / \mathrm{PR}$ for the $R, \theta_{x u}$ and $\theta_{z u}$ refer to the (110) face, and for the $R^{\prime}, \theta^{\prime}{ }_{x u}$ and $\theta_{z u}^{\prime}$ are the values for the (110) face. Some combinations of $\theta_{x u}$ and $\theta_{z u}$ can be eliminated because $x$ relative to $s$ is known from the crystal structure. (The treatment assumes that the direction of $x$ in the long wavelength tail is unchanged from that at the $445-\mathrm{nm}$ maximum.) The values of $\theta_{s u}$ and $\theta_{x u}$ underlined in Table IV correspond to the assignments of $s$ on the basis of interedge angles and extinction directions. They yield a ratio of 0.48 for the right-hand side of the equation $\left(\theta_{z u}=\theta_{s u}\right)$, to be compared with the left-hand side ratios of 1.27 for the $x$ orientation of Eaton et al. (1975) and Yu et al. (1976) and -3.1 for the $x$ orientation of Johansson et al. (1979). One can also calculate $\epsilon_{z} / \epsilon_{x}$, the ratio of the extinction coefficients of the two transitions, for each face. The $x$ orientation of Eaton et al. (1975) again gives the most consistent values, with $\epsilon_{z} / \epsilon_{x}$ at $560 \mathrm{~nm}$ equal to 2.4 and 6.2 for the (110) and (110) faces, respectively.

\section{Discussion}

In numerous model and flavoprotein complexes, the observed long wavelength absorbance has been attributed to charge transfer between stacked donors and acceptors, but the orientation of the transition moment for the low-energy absorption has not been determined experimentally. Powder spectra have been measured for riboflavin-quinone complexes (Fleischman \& Tollin, 1965). But for most of the crystalline model complexes, only qualitative indications of spectral properties have been recorded: crystals are stated to be red or red-orange (Fritchie \& Johnston, 1975; von Glehn et al., 1970; Scarbrough et al., 1976) and black (Tillberg \& Norrestam, 1972) or green

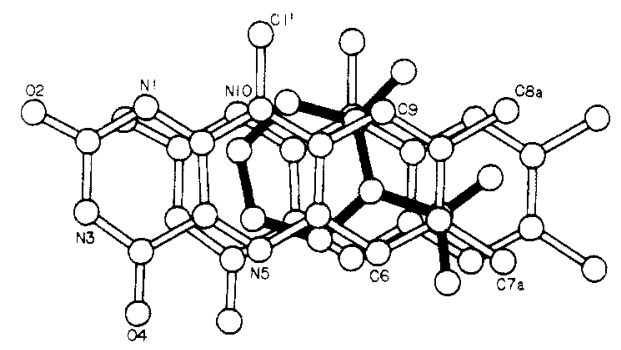

FIGURE 7: Projection of three consecutive layers of the complex lumiflavin-2-aminobenzoic acid onto the plane of the isoalloxazine ring, illustrating the stacking relationships in the structure. The central aminobenzoic acid is drawn with filled bonds; the lumiflavin at the rear is related to that in front by a unit cell translation in $c$; atoms of the rear molecule can thus be identified by comparison with the frontmost molecule.

(Karlsson, 1972). Although it has seemed reasonable to assume that these low frequency bands arise from charge transfer in the stacking direction, all the complexes involving aromatic ligands display in-plane as well as stacking interactions. In crystals of a flavin-nicotinamide model system, in which a reduced flavin is covalently linked to position $N(10)$ via a three-carbon bridge (Porter et al., 1977), the origin of the red color is particularly puzzling since the only stacking interactions involve pairs of bent reduced flavin rings which are not in unusually close contact. We therefore considered it important to establish the transition moment direction for the long wavelength absorbance in crystalline lumiflavin-2aminobenzoic acid.

The polarized single crystal absorption spectra reported here show that the absorbance at wavelengths greater than $530 \mathrm{~nm}$ contains a component which cannot be attributed to transitions in the plane of the flavin ring. The out-of-plane absorbance must therefore arise from a charge-transfer interaction between stacked lumiflavin and aminobenzoic acid molecules. The assumption that both in-plane and out-of-plane (perpendicular) transitions overlap in this spectral region yields reasonable agreement with the data. Two stacking modes occur in lumiflavin-2-aminobenzoic acid crystals (Figures 6 and 7). The spectroscopic measurements do not enable the assignment of the charge-transfer interaction to one or the other of these modes; indeed, both may contribute to the absorbance.

A unique portion of the lumiflavin-2-aminobenzoic acid stacking structure is illustrated in Figure 7, which shows three layers, with the 2-aminobenzoic acid molecule in the middle. The dimethylbenzene and pyrazine rings of the upper (front) lumiflavin of Figure 7 overlap the central aminobenzoic acid, which in turn overlaps all three rings of the lower (rear) lumiflavin. The stacking distance, or average perpendicular distance of the atoms in one layer to the least-squares plane of the molecule in the adjacent layer, is $3.360 \AA$ for the lower (rear) pair and $3.419 \AA$ for the upper (front) pair, suggesting slightly stronger interaction between the lower molecules. Although Yagi et al. $(1968,1970)$ and Massey \& Ganther (1965) have suggested the direct participation of the nitrogen atom of 2-aminobenzoate in the charge-transfer interactions with the isoalloxazine ring system of D-amino acid oxidase, such participation is not evident in the present structure. As shown by Figure 7, the amino group is out of the stacking region of the isoalloxazine ring. The nearest neighbors of the amino nitrogen are $C(9)$ (front) at $3.56 \AA, N(10)$ (rear) at $3.59 \AA$, and $\mathrm{Cl}^{\prime}$ (rear) at $3.52 \AA$. Thus the charge-transfer interaction probably involves the whole delocalized $\pi$ system of the electron-rich 2-aminobenzoate.

Short interplanar spacings and close approach by at least some of the donor-acceptor atoms have often been observed 
in presumed charge-transfer complexes of flavins, but the upper limit for contacts involving charge transfer is not well-defined. Wells et al. (1974) attribute the absence of pronounced long wavelength absorbance in the anhydrous complex of 2,3naphthalenediol-lumiflavin to the lengths of the interplanar spacings, which are 3.41 and $3.48 \AA$; the trihydrate form of the same complex forms red-orange crystals with spacings of 3.11 and $3.26 \AA$. On the other hand, lumiflavinium cationhydroquinone forms dark green crystals in which stacking involves only the benzenoid portion of the flavin, with an interplanar spacing of $3.49 \AA$. The stacking and contact distances are not dramatically short in lumiflavin-2-aminobenzoic acid. The smaller of the stacking spacings, $3.360 \AA$, is slightly less than the usually quoted van der Waals distance for aromatic groups. Three intermolecular atom-atom contacts in the structure are found to be less than $3.40 \AA$. They are $\mathrm{C}(7)$ of aminobenzoic acid to lumiflavin $\mathrm{C}(7)$ (front in Figure 7), $3.371 \AA ; C(6)$ of aminobenzoic acid to lumiflavin $\mathrm{C}(4 \mathrm{a})$ (rear), $3.396 \AA$; and $C(2)$ of aminobenzoic acid to lumiflavin $\mathrm{N}(10)$ (rear), $3.362 \AA$.

A survey of the known structures of stacked "colored" flavin complexes reveals a variety of azimuthal and translational orientations of the ligand with respect to the isoalloxazine framework. The range of permitted orientations, within the broad requirement of extensive aromatic overlap, has been noted previously (Fritchie \& Johnston, 1975). For example, the relations of the hexagonal nets of donor and acceptor are not constant from one structure to another. In lumiflavin2-aminobenzoic acid, the donor and acceptor ring systems are rotated by an angle of approximately $15^{\circ}$. A similar angle has been found in lumiflavinium bromide-sesqui(naphthalene-2,7-diol) monohydrate (Langhoff \& Fritchie, 1970), lumiflavinium chloride-hydroquinone (Karlsson, 1972), and lumiflavinium bromide-hydroquinone (Tillberg \& Norrestam, 1972). On the other hand, isoalloxazine and ligand nets are almost exactly parallel in lumiflavin-diaminopurine (Scarbrough et al., 1976) and 10-propylisoalloxazine-bis(naphthalene-2,3-diol) (Kuo et al., 1974). These variable geometries are consistent with the usual assumption that charge-transfer forces are weak and do not contribute to a major extent to the ground-state interactions (Mayoh \& Pront, 1972). Instead, the mutual orientation of the aromatic planes is controlled by the usual dispersion, coulomb, and hydrogen-bonding interactions, and it seems reasonable to assume that the position of the ligand can be influenced by its in-plane interactions in the crystalline state.

From comparisons with the lumiflavin-2-aminobenzoate complex, it is tempting to conclude that the 1:1 complex of D-amino acid oxidase with 2-aminobenzoate, which has been considered a typical flavoprotein charge-transfer complex (Massey \& Ghisla, 1974), also involves stacking of the ligand over the isoalloxazine chromophore with considerable $\pi$ overlap. While the latter assumption is reasonable, no predictions should be made from model crystal structures with respect to the precise azimuthal and translational orientation of prosthetic group and substrate or inhibitor in the enzyme complexes. As noted by Slifkin (1971), a charge-transfer transition can be highly dependent not only on the reciprocal orientation of the partners in a complex but also on the environment, e.g., the presence of charges at the enzyme active center. Specific protein-ligand interactions must be responsible for the somewhat puzzling observation that the complex of D-amino acid oxidase with $p$-aminobenzoate, which should be as good a donor as 2-aminobenzoate, does not display any long wavelength absorption (Massey \& Ganther, 1975).

\section{Acknowledgments}

L.K.H. thanks Dr. W. A. Eaton of the National Institutes of Health for use of the microspectrophotometer and Dr. J. C. Sutherland of Brookhaven National Laboratory for his assistance with the powder spectra. Initial studies of the crystal spectra were performed while L.K.H. was a member of the Laboratory of Chemical Physics, National Institute of Arthritis, Metabolism and Digestive Diseases. We are grateful to Katherine A. Pattridge for assistance in indexing the crystal faces.

\section{Supplementary Material Available}

A listing of observed and calculated structure factors (9 pages). Ordering information is given on any current masthead page.

\section{References}

Abramovitz, A. S., \& Massey, V. (1976) J. Biol. Chem. 251, 5327-5336.

Bear, C. A., Waters, J. M., \& Waters, T. N. (1970) J. Chem. Soc. $D, 702$.

Boone, C. D. G., Derissen, J. L., \& Schoone, J. C. (1977) Acta Crystallogr., Sect. B B33, 3205-3206.

Brown, C. J. (1968) Proc. R. Soc. London, Sect. A A302, 185-199.

Cohn, E. J., \& Edsall, J. T. (1943) in Proteins, Amino Acids and Peptides, pp 127-128, Reinhold, New York.

Darling, G. D. (1975) Ph.D. Dissertation, The University of Michigan, Ann Arbor, MI.

Dolin, M. (1957) J. Biol. Chem. 225, 557-573.

Eaton, W. A., Hofrichter, J., Makinen, M. W., Anderson, R. D., \& Ludwig, M. L. (1975) Biochemistry 14, 2146-2151.

Fleischman, D. E., \& Tollin, G. (1965) Biochim. Biophys. Acta 94, 248-257.

Foster, R. (1969) in Organic Charge Transfer Complexes (Bloomquist, A. T., Ed.) pp 33-71, Academic Press, New York.

Fritchie, C. J., Jr., \& Johnston, R. M. (1975) Acta Crystallogr., Sect. B B31, 454-461.

Hemmerich, P., \& Schuman-Jorns, M. (1973) in Enzymes: Structure and Function (Drenth, J., Oosterbaan, R. D., \& Veeger, C., Eds.) p 95, North Holland, Amsterdam.

Hofrichter, J. (1979) J. Mol. Biol. 128, 335-369.

Hofrichter, J., \& Eaton, W. A. (1976) Annu. Rev. Biophys. Bioeng. 5, 511-560.

Inoue, M., Shibata, M., \& Ishida T. (1980) Biochem. Biophys. Res. Commun. 93, 415-419.

Johansson, L. B.-Å., Davidsson, Å., Lindbolm, G., \& Naqvi, K. R. (1979) Biochemistry 18, 4249-4253.

Karlsson, R. (1972) Acta. Crystallogr., Sect. B B28, 2358-2364.

Kierkegaard, P., Norrestam, R., Werner, P.-E., Csöregh, I., von Glehn, M., Karlsson, R., Leijonnarck, M., Rönnquist, O., Stensland, B., Tillberg, O., \& Törbjornsson, L. (1971) Flavins Flavoproteins, Proc. Int. Symp. 3rd, 1-22.

Kuo, M. C., Dunn, J. B. R., \& Fritchie, C. J., Jr. (1974) Acta Crystallogr., Sect. B B30, 1766-1771.

Langhoff, C. A., \& Fritchie, C. J., Jr. (1970) J. Chem. Soc. D, 20-21.

Lockridge, O., Massey, V., \& Sullivan, P. A. (1972) J. Biol. Chem. 247, 8097-8106.

Main, P., Woolfson, M. M., Lessinger, L., Germain, G., \& Declercq, J. P. (1974) MULTAN '74. A System of Computer Programmes for the Automatic Solution of Crystal Structures from X-ray Diffraction Data. 
Massey, V., \& Palmer, G. (1962) J. Biol. Chem. 237, 2347-2358.

Massey, V., \& Gibson, Q. H. (1964) Fed. Proc., Fed. Am. Soc. Exp. Biol. 23, 18-29.

Massey, V., \& Ganther, H. (1965) Biochemistry 4, 1161-1173.

Massey, V., \& Ghisla, S. (1974) Ann. N.Y. Acad. Sci. 227, 446-465.

Massey, V., Matthews, R. G., Foust, G. P., Howell, L. G., Williams, C. H., Zanetti, G., \& Ronchi, S. (1970) in Pyridine Nucleotide-Dependent Dehydrogenases (Sund, H., Ed.) pp 393-311, Springer-Verlag, Berlin.

Mayoh, B., \& Pront, C. K. (1972) J. Chem. Soc., Faraday Trans. 2, 1072-1082.

Norrestam, R., \& Stensland, B. (1972) Acta Crystallogr., Sect. B B28, 440-447.

Porter, D. J. T., Bright, H. J., \& Voet, D. (1977) Nature (London) 269, 213-217.

Scarbrough, F. E., Shieh, H.-S., \& Voet, D. (1976) Proc. Natl. Acad. Sci. U.S.A. 73, 3807-3811.

Scarbrough, F. E., Shieh, H.-S., \& Voet, D. (1977) Acta Crystallogr., Sect. B B33, 2512-2523.

Slifkin, M. A. (1971) in Charge Transfer Interactions of Biomolecules pp 132-171, Academic Press, New York.

Sutherland, J. C., \& Boles, T. T. (1978) Rev. Sci. Instrum. $49,853-857$.
Sutherland, J. C., Desmond, E. J., \& Takacs, P. Z. (1980) Nucl. Instrum. Methods, 172, 195-199.

Strickland, S., \& Massey, V. (1973) J. Biol. Chem. 248, 2953-2962.

Tillberg, O., \& Norrestam, R. (1972) Acta Crystallogr., Sect. B B28, 890-898.

Tollin, G. (1968) in Molecular Associations in Biology (Pullman, B., Ed.) pp 393-409, Academic Press, New York.

Trus, B. L., Wells, J. L., Johnston, R. M., Fritchie, C. J., Jr., \& Marsh, R. E. (1971) J. Chem. Soc. D, 751-752.

von Glehn, M., Kierkegaard, P., Norrestam, R., Rönnquist, O., \& Werner, P.-E. (1970) Acta Chem. Scand. 24, 3701-3710.

Wang, M., \& Fritchie, C. L., Jr. (1973) Acta Crystallogr., Sect. B B29, 2040-2045.

Wells, J. L., Trus, B. L., Johnston, R. M., Marsh, R. E., \& Fritchie, C. J., Jr. (1974) Acta Crystallogr., Sect. B B30, 1127-1134.

Yagi, K., Okamura, K., Naoi, M., Sugiura, N., \& Kotaki, A. (1967) Biochim. Biophys. Acta 146, 77-90.

Yagi, K., Ozawa, T., Naoi, M., \& Kotaki, A. (1968) Flavins Flavoproteins, Proc. Conf., 2nd, 237-251.

Yagi, R., Naoi, M., Nishikimi, M., \& Kotaki, A. (1970) J. Biochem. (Tokyo) 68, 293-301.

Yu, M. W., Fritchie, Jr., Fucaloro, A. Fl, \& Anex, B. J. (1976) J. Chem. Soc. 97, 6496-6500. 